\title{
Nuevos realismos y sociedades complejas
}

\author{
Mario Samaniego Sastre
}

\section{Resumen}

Las relaciones entre ciencia y sociedades complejas conforman el trasfondo de este texto, en el cual se reflexiona sobre el diálogo, tensión o escisión entre la ciencia entendida ya como dominio de lo público, como bien público y la sociedad civil en la que conviven grupos e individuos con identidades y concepciones de vida diversas e incluso confrontadas. Para ello, se describen ciertos hitos que dan cuenta de los cambios que tanto al interior de la epistemología, filosofía y teoría social como en la vida se han ido gestando en la modernidad tardía, los cuales al menos potencialmente abocan los esfuerzos tanto en el plano teórico como en el social a trabajar en pos del diálogo. Sin embargo, pareciera, que existiendo la oportunidad de establecer una relación deliberativa entre ciencia y sociedad, ésta por el momento no se da.

\begin{abstract}
The background of this text is given by the relationships between science and complex societies; it offers an analysis about the dialogue, the tension or the division between science-already understood as a public good-and civil society, in which both groups and individuals with diverse identities and conceptions of life-even confronted ones- coexist. Some benchmarks are described which give account of the changes that have been generated both inside epistemology and philosophy and also in life and social theory in late modernity, which at least potentially address their efforts both from the theoretic and the social scenarios to work in favor of the dialogue. Nevertheless, it seems that even though the opportunity to establish a deliberative relationship between science and society does exist, at the time being it has not occurred.
\end{abstract}

\section{Sociedades complejas y globalización}

U n sistema complejo puede ser caracterizado de forma abstracta y sucinta por la ausencia de objetos y/o par tes, además de carecer de límites. Por el contrario, sería una red siempre constituyéndose en virtud de relación de relaciones dinámicas entre sus nudos. Nuestras actuales sociedades parecieran que se adecuan en sus fisonomías a un sistema complejo. La tradicional distinción entre sociedades tradicionales y sociedades modernas quedaría limitada a la hora de proporcionar claves analíticas para explicar y/o comprender las prácticas de los actuales sujetos, organizaciones e instituciones. Siguiendo una interpretación posible entre otras ${ }^{2}$, una sociedad tradicional es aquella que se constituye a partir de un fundamento sacral. El surgimiento de las sociedades modernas, en tanto sociedades diversas en sus fundamentos, se alejaron del fundamento sagrado, organizándose en este caso desde el impacto de la industrialización y el interés ilustrado de buscar una autoorganización social basada en la moral, moral humana desligada de cualquier raigambre religiosa. El impacto de la industrialización no interesa tanto verlo en su dimensión material, el hecho en sí de la emergencia de nuevas formas de producción, cuando en la esfera ideacional y en último término ideológica que éstas trajeron consigo, debiéndose organizar la sociedad a partir de los ideales de la economía política. Esto en principio, proporcionaría un desarrollo que redundaría en un bienestar material para los ciudadanos. La moral, perseguiría configurar modelos de organización decididos y pactados por los mismos ciudadanos, según los dictados de su razón, los cuales procurarían desarrollo ya no material, sino podríamos decir espiritual. El resultado de este esfuerzo, nos parece, desembocó en instituciones y discursos universales y abstractos, entendido esto último como construcción al margen de los intereses de los individuos y los grupos, al no erigirse tales discursos

1 Filósofo. Acadẻmico Escuela de Antropología. Universidad Católica de Temuco

2 Referida a partir de los textos de Habermas previos a la publicación de la Teoría de la Acción Comunicativa. 
desde las formas de buena vida que ellos consideran como tales. Así, el estado nación, la declaración de los derechos del ciudadano de la Bastilla o la Declaración Universal de los Derechos Humanos de 1948, son algunos ejemplos ${ }^{3}$. Lo anterior no quiere decir que en nuestras sociedades no existan sociedades tradicionales, ni estén vigentes aunque en crisis o con acecho de ésta, dimensiones de las sociedades modernas. Sólo queremos apuntar a que los rasgos indicados son insuficientes para entendernos societalmente hoy.

Bajo las sociedades tradicionales o modernas subyace la idea de orden. Nada en ellas quedaría al amparo de la locura o el caos. Fueran los fundamentos sagrados, las impersonales leyes de la economía política o los pactos logrados a veces incluso con sangre, éstos regulan monológicamente desde los límites que ellos mismos marcan, las prácticas sociales en un intento de orientar a los grupos humanos hacia un destino común, presente ya en el mismo fundamento. Toda escatología exige una genealogía ${ }^{4}$.

A diferencia de lo indicado, nuestra sociedad sería una sociedad red ${ }^{5}$, red dinamizada por una dialéctica orden-desorden ${ }^{6}$. Vivimos en la sociedad de conocimiento; el conocimiento, el acceso al mismo y su producción, son condiciones necesarias para que los sujetos puedan no quedar excluidos de los beneficios de los sistemas. El conocimiento está indisolublemente ligado a la justicia social, y hoy vemos como los conocimientos se organizan, distribuyen, se concentran o dispersan de manera virtual, a través de las diversas redes de información y asociaciones internacionales, a las cuales no todos tienen acceso. Otros rasgos de nuestra sociedad en la perspectiva de la red se manifestarían en el debilitamiento del estado-nación, siendo en la práctica cada vez más, una red de naturaleza supraestatal la que toma las decisiones que afectan a los ciudadanos de un estado particular. El estado se queda sin límites definidos, se reconstruye en las diversas redes a las que continuamente se va integrando, siendo igualmente su actuar influenciado por movimientos que hasta hace poco le eran ajenos, por ejemplo las ONG's. Vemos de este modo, que entender el estado exige inmiscuirse en la red de relaciones de las que forma parte y en las que él mismo influye, como medio para poder entender su actuar. La trasnacionalización y concentración del poder económico e ideológico es otro aspecto que inevitablemente nos sitúa en una perspectiva relacional. Quizá como consecuencia de esto, es que cada vez con mayor notoriedad surgen nuevos agentes sociales y políticos que luchan por el reconocimiento cultural de nuevas identidades e identidades invisibilizadas y/o reprimidas, quedándose su acción no anclada en la necesidad de reconocimiento, sino en la posibilidad de decidir autónomamente en los asuntos de naturaleza diversa que les competen o afectan, recursos, patrimonios, modelos de organización política, marco jurídico positivizado, etc. Y todo esto, más allá o quizá por lo mismo, de la mano de la globalización. Globalización que en principio pareciera que sería un destino fatal frente al que no hubiera alternativa y que redundaría en una disolución de identidades conformando un nuevo mapa geopolítico y humano homogéneo. Sin, embargo, este supuesto destino fatal, no se manifiesta con claridad. Por el contrario, las particulares proyecciones de los individuos y grupos permanecen, eso sí, sometidos a la incertidumbre, a riesgos, los cuales pueden recorrer una gama variada de posibilidades, siendo las más extremas y menos deseables, aquellas que podemos denominar clausura radical, por ejem$\mathrm{plo}$, nacionalismos y culturalismos fundamentalistas y por otro lado, las exteriorizaciones diluyentes, esto es, pérdida de ethos, deriva, inercia.

Cualquiera de los casos o situaciones planteadas, aunque diversas y discutible, nos indican que, a diferencia de los que sucedía-sucede en las sociedades tradicionales y modernas, una lógica del no equilibrio, de la incertidumbre las recorre, siendo el futuro abierto y riesgoso. La globalización va de la mano de la complejidad. Esta nueva sociedad, es más pensable desde la crisis de los fundamentos que social y teóricamente, tradiciones muy variadas pero convergentes en la idea de orden han postulado, desarrollado y mantenido. La dialéctica orden-desorden como proceso es manifiesta. Así los eventos lo podemos identificar con dispersión, choque, los cuales sólo pueden ser entendidos en su vinculación, en la inestable diada orden-desorden.

\section{Epistemología e identidades complejas}

En este estado de cosas, los estudios científicos sobre procesos socioculturales tendrían que contemplar el nuevo escenario al cual pertenecen, mirar-actuar en él, desde él mismo; la epistemología clásica se torna limitada, insuficiente e improcedente. Además de los procesos sociales y culturales reseñados anteriormente, surgieron desde finales del siglo $\mathrm{XIX}$, desarrollos científicos que socavaron las bases de la atemporalidad, idealización (como desarraigado de las diferencias socioculturales) y homogeneización que exigen los principios de la epistemología clásica. Así, por citar algunos, el neopositivismo lógico (con su interés en las estructuras abstractas e intemporales, la lógica de la ciencia, los enunciados lógico-matemáticos a priori, como instrumento de análisis de lo sintético y a posteriori, fundado en la experiencia), comienza ya en 1931 a tener críticas demoledoras: Gödel demuestra las graves limitaciones de toda formalización, aún restringiéndose a la teoría de los números. Después vendría Quine (1951) rechazando la distinción entre lo analítico y sintético, al igual que el reduccionismo a la experiencia, Wittgenstein (1953) ataca dos supuestos fundamentales del positivismo, los enunciados atómicos de base y la limi-

\footnotetext{
3 En principlo se aceptarfa una Interpretaclón neutral de los mismos

4 Cf. Lyotard, J.F. (1986) La condición posmoderna, Cátedra, Madrid

5 Para una visión desde el punto de vista de la socledad de la información, nos remitimos a Castells, M. (1997) La era de la información, Alianza, Madrid

6 Siguiendo a Morin (1996) Ciencia con Consciencia, Alianza, Madrid
} 
tación del lenguaje a aseveraciones analizables en término de juntores de verdad. Hanson, Toulmin, Feyerabend y otros, terminan demostrando que esta concepción no se ajustaba a la praxis histórica y real científica, mostrando su dogmatismo y apriorismo. Las diferentes interpretaciones que surgen sobre probabilidad e inducción, muestran la débil vinculación de nuestro conocimiento con el mundo de los hechos, la isomorfía desaparece, la problemática de la adecuación y el realismo mitigado se extrema con la irrupción de la mecánica cuántica. Estos acontecimientos, unidos al nuevo paisaje sociocultural, fueron dando lugar a un giro epistémico, alguna de cuyas características podrían ser: La nueva inducción, probabilística y con explicación estadística, el pluralismo teórico, las transformaciones paradigmáticas o epistémicas, aceptación de que diversas legalidades subyacen al conocimiento científico, aceptación igualmente de la activa función del sujeto en la observación científi$\mathrm{ca}$, necesidad de la epistemología y hermenéutica como instancias requeridas para un acercamiento comprensivo de la ciencia, existencia de la complejidad en la materia y en el papel lógico-organizador del sujeto, en último término, incertidumbre. De este modo, el mundo es nuestra observación, no es plausible zafarse de lo que implica la ontología del observador. El ojo está ahí como reflejo, como opacidad, como reinterpretación ${ }^{7}$.

Se podría aceptar ya, que el conocimiento de los otros, de la realidad (disposición ante ella, ellos) dista mucho de ser un proceso de transparentación, aclaramiento de la realidad. Por el contrario, las perturbaciones están tanto en el sujeto como en el objeto (el otro sujeto) de conocimiento e interacción. Es más, el acoplamiento entre ambos se lleva a cabo en las perturbaciones que mutuamente se infringen. La perturbación es transversal al conocimiento e interacción. Por lo mismo, se debería negar tanto el realismo ingenuo y imitigado?, rechazar la posibilidad de aceptar que el mundo es como lo que vemos, al igual que el azar sin más. Ambas posiciones terminan justificándose en el fundamentalismo. De este modo es necesario el alejamiento de la objetividad, la cual determina un sujeto desajustado, un "se" impersonal. Por tanto es perentorio, anularse como sujeto y aceptarse como observador situado.

La objetividad fue propia de la ciencia nueva, moderna, ciencia que se dedicó a modelar matematizando, a catalogar y ordenar, ciencia que nunca fue capaz de ser caja de resonancia de los eventos, de los acontecimientos. Ello, porque las teorías y leyes eran de naturaleza reversible, y en lo reversible quedan siempre fuera los acontecimientos en su totalidad y complejidad, ya que sólo se consideran algunos elementos que son categorizados como modélicos. En realidad, la ciencia nueva o moderna redujo lo múltiple a lo uno ${ }^{8}$. Y sin embargo, los acontecimientos son irreversibles, 10 que nos interpelaría a vivenciar la irreversibilidad de nuestra temporalidad como sujetos socioculturales y científicos ¿Qué posibilidades se dan para integrar al acontecimiento-proceso en nuestro discurso? Emerge el observador: Este "crea un mundo" que siempre lo manipula a través de reducciones simplificadoras. La existencia de los particulares hombres y grupos humanos influyen en el mundo para que tengamos el que tenemos entre todos los mundos posibles. El observador constriñe, ya que otros mundos serían posibles en otras condiciones de observación ¿Por qué lo crea? Un acontecimiento existe, es, si cumple, si se ajusta a principios que este observador configura a partir de un marco de referencia que permite la existencia de ciertas posibilidades, que adquieren existencia real, precisamente porque no son capaces de escaparse del marco y los principios. Hemos de advertir que siempre estamos enfrentados a acontecimientos reales, aquellos que se ajustan a los principios, pero que también existen otros posibles. "Definitivamente, no podemos seguir relacionándonos con el mundo como si la realidad tuviera una existencia de objeto, de algo externo a nosotros como conocedores, independiente del acto mismo por el cual la conocemos. No es necesario que neguemos esa existencia objetiva de lo real: nada nos obliga a llegar hasta ese punto; pero los elementos de que disponemos nos obligan, sí, a poner entre paréntesis esa objetividad, y a proceder en consecuencia. Más aún: hemos de reconocer que la naturaleza elusiva de lo real, su indeterminación, no es solamente una condición otorgada por el observador, sino que propia de la physis misma. La realidad aparece, en su condición misma, como indeterminada, incierta, en cambio, ambivalente, puesta en escena: enactuada en el proceso mismo de nuestro relacionarnos cognitivamente. De un modo a veces inexplicable, participamos en la producción de lo que constituye nuestra realidad: nada hay que quede fuera de nuestra mente corporizada".(Gutiérrez, 2001:13)9

El giro epistémico descrito se podría entender como una superación de la epistemología al alejarse de todo fundamentalismo gnoseológico y metafísico, al intentar evitar la vocación cartesiana de buscar un punto fijo e indubitable, además de legitimador, como mecanismo para evitar el desorden. Frente a lo gnoseologización del pensamiento mođerno, parecieran más adecuados a la realidad sociocultural en la que nos desenvolvemos, situarse en los denominados giro ontológico, tal como Gadamer $^{10}$ lo lleva a cabo, y/o giro lingüístico en sus diversas variantes, Habermas ${ }^{11}$ por ejemplo, para que el otro aparezca en nuestro horizontes y esté a nuestra mano. Y este es el gran problema; hasta este momento, el otro, ese otro complejo y situados en estructuras disipantes, no había aparecido. La gnoseologización moderna impidió tal posibilidad, invisibilizó todo aquello situado más allá de los límites de un tiempo, un espacio y un tipo de legalidad sociocultural que se delineaba desde sí mismo, desde sus propios fundamentos. La tradición

\footnotetext{
7 En esta idea convergen autores que podemos aglutinar en la denominada ontología del observador, el constructivismo y las implicancias epistémicas derivadas de la nueva biología 8 Esta reducción tiene repercusiones no sólo en el plano epistémico, sino también en el ontológico y ético. A modo de ejemplo, las tesis de Vattimo y Levinas

9 La cita es de un texto Inédito. Conferencia dictada en Temuco, Chile en el año 2001

10 En su pensamlento se presenta la universalización de la interpretación como condición de la facticidad. Véase GADAMER H.G. (1982) Verdad y Método, Ed. Sigueme, Salamanca

11 Sobre la reformulación dialógica del imperativo categórico en la ética discursiva, véase HABERMAS J (1999) La inclusión del otro, Paidós, Barcelona
} 
platónica al concebir el ser como identidad consigo mismo, concepción que dio paso a toda una tradición ética, ontológica y epistemológica a la medida de tal referente en diversas variantes, cegó el encuentro con ese otro diverso y complejo, e impidió construir fundamentos constituyentes de relaciones dialógicas con lo otro.

En esta nueva coyuntura, hay que saberse situados siempre en la incertidumbre, en el riesgo, debiendo evitar en términos de Nietzsche el consuelo de la metafísica. Deshacerse de la idea de fundamentos indubitables, donde los entes nos hablarían de los diferentes órdenes existentes, pero bajo los cuales subyacería un fundamente (ser) atemporal. Este giro epistémico implica el que el científico sociocultural adquiera unas actitudes y hábitos, además de ciertos valores de tal naturaleza que, la tradicional afirmación según la cual, los únicos valores de la ciencia son los de la epistemología, sea una afirmación a desterrar de las comunidades científicas. De este modo, el falabilismo ${ }^{12}$, como capacidad para estar abierto siempre a la crítica, para reformularse, si bien es categoría epistémica, de igual manera podría convertirse en un hábito. Aún más, este hábito no tendría por qué sorprender, dada la compleja contingencia histórico social. Ésta hace que nos movamos entre la tragedia y la esperanza, donde el error será dimensión inherente a las prácticas científicas, quizá incluso pudiendo ser visto como necesario, ya que obliga a abrir nuevas vías, a ser creativos para proponer nuevos horizontes de posibilidad. Otra actitud, que se podría resaltar desde el pensamiento de Gadamer, es la limitación en la que el sujeto histórico y también el científico, padecen, limitación virtuosa por cuanto la condición interpretativa es radicalmente abierta. Esto nos habla de limitación, limitación por cuanto como interpretador, no se puede saber con certeza de la realidad, pero paralelamente, la limitación es fuente de apertura, que permite y posibilita la fusión de horizontes culturales; esto es, el encuentro con el otro en un trasfondo históricamente situado, evitando así, una de las más perniciosas consecuencias de las prácticas científico-sociales en el marco de la epistemología clásica, reducir el otro a lo uno, representar al otro desde los parámetros de uno, los cuales a su vez, se legitiman en fundamentos articulados desde esa lógica platónica de identidad consigo mismo. Desde nuestro punto de vista esto es decisivo, ya que no se ha de olvidar que es la cotidianidad el punto de partida para entender a las personas, no son las teorías ni los fundamentos que las sustentan, las que permiten la comprensión del mundo, sino el asumir esa cotidianidad. Se estaría en el paso de una gnoseologización de la realidad a una ontología de la facticidad ${ }^{13}$. Esto nos llevaría a aceptar la teoría como interpretación, que en cuanto tal, no puede rehuir el diálogo, frente a la teoría como universalidad desconectada de los mundos de la vida. La verdad, meta suprema y producto de la representación realista de una epistemología fundada en una separación sujeto-objeto, en una metafísica de la subjetividad, ya no es básicamente ejercicio de la razón, tampoco consenso, ni meta a alcan- zar, sería proceso dialógico. Más allá del pensamiento de la subjetividad moderna, pensamiento de la intersubjetividad, pensamiento como diálogo. Surgen nuevas implicancias que exigen nuevas actitudes. La reflexión, según lo anterior, operaría como un salir fuera de sí, conectarse con lo extraño y recogerse en sí mismo renovado. Pudiera pensarse que con la lógica de la intersubjetividad pudiera ocurrir algo similar a lo que se indicó al referirse al pensamiento de la subjetividad, el que lo otro se reduzca a lo uno, el que en esa salida dialógica fuera de sí, el uno sea engullido por lo otro. Esto no tendría por qué ocurrir, ya que la dialogía intersubjetiva no está abocada al logro de consensos, sino dado que lo que en él prima son las identidades y no los acuerdos. Incluso más, antes referíamos la necesidad del error; en este caso se apostaría a que enfrentamientos dialógicos con concordia y dignidad, permitirían avances, desarrollos y generación de nuevas hipótesis.

\section{Tensiones entre ciencia y sociedad}

Hasta aquí hemos dado cuenta de los giros epistémico producto del nuevo paisaje sociocultural como consecuencia de la complejización de las sociedades modernas y de la autoreflexión que la misma epistemología ha desarrollado a partir de procesos y acontecimientos científicos que mostraron inconsistencias y limitaciones en sus mismos fundamentos. De todo ello, se han inferido nuevas categorías epistémicas vinculadas para su materialización a valores y actitudes muy diversos a los de la propia epistemología clásica, tales como exactitud, rigor, verdad como coherencia, etc.

Pero creemos que el problema va más allá si nos preguntamos cómo efectivamente funciona hoy la ciencia y la tecnología; es decir, más allá de criterios y reflexiones epistemológicas, filosóficas y socioculturales, aunque obviamente éstas también operen, la pregunta acerca de cómo se vinculan la ciencia y la tecnología con la sociedad, cómo actúan en ella, merece una reflexión aparte. Y esta reflexión se entiende desde una asimetría que da cuenta de la complejidad de nuestras sociedades en las relaciones entre ciencia y sociedad ${ }^{14}$. Por una parte, la epistemología ha reflexionado y tomado conciencia de la necesidad de no dejar fuera al otro en sus procesos de conocimiento; en la misma línea, es notoria la importancia que el diálogo está tomando en nuestra sociedad plural como condición para una convivencia armónica, habida cuenta de que pluralismo y complejidad son dimensiones estructurales y patentes de la misma, y sin embargo las relaciones ciencia sociedad no pueden ser entendidas bajo el parámetro dialógico indicado.

Podríamos decir que hasta no hace mucho tiempo, las prácticas cientifficas y tecnológicas dependían de las propias comunidades científicas sobre la base de sus propios intereses. Hoy por el contrario la situación es bien diversa. Las ciencias y las tec-

12 En este caso lo interpretamos tal como la tradición pragmatista.

13 En el desarrollo posmetafisico de Heidegger en HEIDEGGER M (1982) Ser y Tiempo, FCE, México

14 A modo de ejemplo, como representativo de una sociología del conocimiento, véase BLOOR D (1998) Conocimiento e imaginario social, Gedisa, Barcelona 
nologías se han convertido en propiedad pública desligada de los intereses presentes en los distintos grupos que conviven en la sociedad civil, produciéndose un conflicto entre lo social y lo científico tecnológico. Además, al no poder participar desde los mundos de la vida la sociedad en las decisiones que competen a la ciencia, en tanto sistema dependiente de una espera distinta, (téngase presente a modo de ejemplo, cómo los grandes fondos concursables en investigación dependen del estado o instituciones trasnacionales) ésta se está convirtiendo en una losa que casi fatalistamente los individuos tienen que soportar, (claro está, nos referimos a sus objetivos, metodologías, etc.), llevando esto a que la ciencia y la tecnología cambien a la gente, incidiendo en su forma de entender el mundo, así como en sus prácticas y objetivos. La tensión entre ciencia y tecnología por una parte y sociedad por otra se manifestaría que las relaciones instrumentales que las primeras tendrían sobre la segunda. En este sentido, pareciera necesaria una relación dialógica entre lo social y lo científico-tecnológico, entre lo público y los mundos privados. Esta relación dialógica se torna necesaria por cuanto incluso se puede producir un conflicto entre verdades científicas, que efectivamente lo sean y concepciones de buena vida de los grupos humanos desde sus mundos de la vida. Si esto no ocurre se cae en el riesgo de que la ciencia y la tecnología con verdades verdaderas se imponga en la sociedad sin legitimación, cooperando poco con ello a la democratización de la misma o a creer que nuestras sociedades lo son, cuando en su seno existen élites cognitivas actuando como enclaves autoritarios. El diálogo en ciencia y sociedad tiene repercusiones que trascienden con mucho los límites de influencia de la epistemología, ya que como decíamos, valores sociales y culturales, que hablan de una buena vida, pueden estar desconectados de los valores con que la ciencia y tecnología actúan, como sistema desligado del ciudadano de a pie, afectándolos y sin que éste pueda tener muchos mecanismos de defensa, entendidos como condiciones para una relación dialógica.

Bajo estas circunstancias, parece que el viejo pero a la vez presente ideal de representación verdadera de la realidad, que el representacionalismo constituido sobre el supuesto de la existencia de lo real desligado de la misma esfera científica y tecnológica, carece de sentido. Por el contrario, las relaciones ciencia y sociedad son analíticamente más visibles sobre la categoría de transformación ${ }^{15}$, es decir, sobre esas transformaciones que de manera lineal y no reflexiva y dialógica, la ciencia va produciendo. Ahora bien, esto no quiere decir que los representacionalismos y realismos hayan dejado de existir. Siguen existiendo pero renovados. Por una parte la representación en la esfera científica no se constituye ya a partir de la conjunción de fundamentos epistémicos e intereses y curiosidades de las comunidades científicas, sino de los intereses del mundo público, denominación que usamos para diferenciarlo de los diversos mundos privados que configuran lo social. Por otra parte, las imágenes y mundo con que los privados articulan sus vidas y proyectos, están siendo trastocados por la influencia de una esfera con la cual el diálogo hoy por hoy se vislumbra lejano. Los estudios especializados sobre los social y cultural, habrán de abocarse a entender la compleja red social sin perder de vista estos nuevos realismos, estos nuevos mundos que se van trastocando producto de la inestabilidad de la red y de la jerarquización no dialogante entre sistemas científico tecnológicos y los propios sistemas de los mundos de la vida donde se tejen las identidades. La transformación nos desafía a que el conocimiento científico incorpore la praxiología.

\section{Conclusiones}

1. Primacía de la tecnociencia y de la filosofía de la actividad científica, que desplazarían a la tecnología y ciencia básica por un lado y a la epistemología por otro. En el primer caso, se estaría constituyendo un nuevo tipo de actividad y conocimiento, caracterizado por ser un conocimiento híbrido que se configura a partir del conocimiento científico, que se constituye a partir de los avances científicos (así por ejemplo, los hechos ya no podrían entenderse como la tradición empirista nos ha trasmitido, sino como resultado de las acciones de la tecnociencia), forjando un entramado complejo que no puede entenderse desde la tradicional distinción entre ciencia y tecnología, dado que el componente axiológico hay que tenerlo necesariamente en cuenta, debido a que este nuevo conocimiento ya no tiene como objetivos las tradicionales propuestas de explicación, predicción, comprensión o interpretación, sino el de transformación, lo que demanda conocer el grado de satisfacción de los valores que están en juego en los indicados procesos de transformación. Estos valores no serían los valores propios de la epistemología, tales como la verdad o exactitud, sino que aparecen nuevos valores producto del eje transformación y del hecho de que las transformaciones involucran actores con referentes valóricos diferenciados e incluso contradictorios, siendo esta situación aún más notoria en sociedades complejas, interétnicas y asimétricas. Lo anterior exige relegar la axiología prescriptivista y abocarse a determinar dialógicamente protocolos de evaluación de las acciones tecnocientíficas, identificando qué valores son realmente significativos y válidos para los actores, siendo además este proceso dinámico, en virtud de la historicidad y la disipante estructura que liga a los actores. Asimismo, la dialogía permitiría transparentar las opacidades tecnocientíficas, las cuales estarían invisibilizando para gran parte de las racionalidades privadas el significado de los productos de la actividad tecnocientífica, y por lo mismo, el sentido de los impactos que éstos producen y que operan con fuerza configurando el tejido sociocultural.

2. Una vez presente en nuestro contexto la cultura tecnocientífica, al menos en el ámbito institucional, hemos de interrogarnos sobre lo siguiente: ${ }$ Es posible aceptar a la tecnociencia en nuevo paradigma de racionalidad? Sería difícil aceptarlo sin que surjan dudas, sobre todo si nos intentamos situar en grupos que operan desde racionalidades simbólicas. Parece poco pertinente y poco eficaz que la epistemología se convierta en criterio para la razón práctica; la ética no debe ser 
gobernada por la racionalidad técnico científica; una cosa es el progreso tecnocientífico y otra el progreso moral. Las identidades que conforman la red de relaciones han de tener mecanismos para decir no frente a algo, quizá frente a imposiciones tecnocientificas no negociadas y por los mismo no validadas. La posibilidad de negarse posibilitaría minimizar el déficit de humanidad que las actividades tecnocientíficas están acarreando en los sistemas socioculturales con referentes valóricos diferenciados respecto de aquellos consensuados por la esfera tecnocientífica.

3. Por lo anterior, todo tipo de conocimiento y también el científico y/o tecnocientífico debería ser conocimiento contextual ${ }^{16}$. La contextualidad ha de ser la dimensión constitutiva y fundante del qué pensamos y cómo lo hacemos; no debe ser un añadido, no debe ser eso que se tiene en cuenta una vez que ya están determinados todas las etapas que conforman las actividades tecnocientíficas, desde la planeación a las transferencias y evaluación. La contextualidad debe ser transversal al proceso. El contexto tiene que ser eje de referencia y espacio donde se debe expresar el conocimiento científico. Concebir las ciencias sociales como actividad que acompaña las prácticas socioculturales, no como instancia que dirige. Concebir la ciencia como acompañamiento dialógico contextual. Las ciencias sociales al servicio de generar dialogo entre contextos, llegando a vislumbrar la posibilidad de considerar el conocimiento como intercontextualidad ${ }^{17}$. Conocimiento que se sitúa en la frontera, frontera como espacio donde surgen las interpelaciones del otro, por tanto no tanto construcción teórica, como caja de resonancia de los ecos que producen las diversidades socioculturales; frontera como convivencia de identidades dinámicas. Por lo mismo el conocimiento no se valida a sí mismo, no puede autofundamentarse, sino en relación a las redes simbólicas en que se inscribe, por lo mismo trasciende los límites de lo cognitivo, ciencia al servicio de descentrar la humanidad, humanidad en que efectivamente puedan resonar las voces de los múltiples rostros que la conforman. Voces que no se organicen a partir de un punto de fuga que unidireccionalmente dinamice la red de relaciones que constituye el tejido sociocultural.

4. La contextualidad antes indicada, sitúa a las ciencias sociales más allá de los límites de los criterios clásicos sobre la base de los cuales se desplegaba el conocimiento científico-social. Así, desde la lógica de la contextualidad el investigador se intenta hacer cargo de los impactos que provoca, exigiendo ello una involucración ética y social con su objeto de estudio; esto es, el investigador no se sustrae de su condición de sujeto sociocultural, aunque claro está, ello no signifique operar desde el plano del sentido común. Bn este sentido parece que sean los grupos involucrados en los procesos de investigación, los que decidan los fines que ésta persiga basándose en los medios de los que se dispone, lo cual requiere procesos de deliberación desde las diferentes racionalidades afectadas; es decir, se superaría la distinción entre el carácter universal de las teorías y las particularidades, siendo en este sentido la teoría una particularidad más, en tanto constriñe a la realidad al reducir su complejidad desde sus propios y finitos referentes. Por el contrario, la apuesta se orienta a la aceptación de competencias diversas, admitiendo la finitud de todas ellas, pero apostando a que cada una posee una posible alternativa que debe ser en principio valorada pasando a nutrir la intercontextualidad.

\section{Bibliografía}

BLOOR D (1998) Conocimiento e imaginario social. Gedisa, Barcelona

CASTELLS M (1997) La era de la información. Alianza, Madrid

FORNET R (2003) Interculturalidad y filosofía en América Latina. Internationale Zeitschrift fur Philosophie, Reihe Monographien. Band 36, Aachen

GADAMER H.G. (1982) Verdad y Método. Ed. Sígueme, Salamanca

HABERMAS J (1987) Teoría de la acción comunicativa. Taurus, Madrid

HABERMAS J (1999) La inclusión del otro. Paidós, Barcelona HEIDEGGER M (1982) Ser y Tiempo. FCE, México

LEVINAS E (1997) Fuera del sujeto. Caparrós, Madrid

LYOTARD JF (1986) La condición posmoderna. Cátedra, Madrid

MORIN E (1996) Ciencia con Consciencia. Alianza, Madrid

VATTIMO G (1985) El fin de la modernidad. Gedisa, Barcelona

16 Usamos la expresión de la filosoiia intercultural acunaada por Raút Fornet Betancourt. Véase FORNET R (2003) Interculturalidad y filosofia en América Latina, Internationale Zeitschrift fur Philosophie, Reihe Monographlen, Band 36 , Aachen

17 Este concepto se entiende como diálogo entre racionalidades plurales e inconclusas 